\title{
The Planck Submillimeter Properties of Galactic High-mass Star-forming Regions: Dust Temperatures, Luminosities, Masses, and Star Formation Efficiency
}

\author{
R. Paladini ${ }^{1}$ (i), J. C. Mottram ${ }^{2}$, M. Veneziani ${ }^{3}$, A. Traficante ${ }^{4}$ (D), E. Schisano ${ }^{4}$, G. Giardino ${ }^{5}$ (iD, E. Falgarone ${ }^{6}$, J. S. Urquhart ${ }^{7}$, \\ D. L. Harrison ${ }^{8,9}$ (i) , G. Joncas ${ }^{10}$, G. Umana ${ }^{11}$, and S. Molinari ${ }^{4}$ (i) \\ ${ }^{1}$ Infrared Processing Center, California Institute of Technology, 1200 E. California Blvd., Pasadena, CA 91125, USA; paladini@ipac.caltech.edu \\ ${ }^{2}$ Max Planck Institute for Astronomy, Konigstuhl 17, D-69117 Heidelberg, Germany \\ ${ }^{3}$ Science \& Technology Corporation, Olof Palmestraat 14, 2616 LR Delft, The Netherlands \\ ${ }^{4}$ IAPS - INAF, Via Fosso Del Cavaliere 100, I-00133, Roma, Italy \\ ${ }^{5}$ ESA, Science Operations Department, ESTEC, 2200AG Noordwijk, The Netherlands \\ ${ }^{6}$ LERMA/LRA, Observatoire de Paris, PSL Research University, CNRS, Sorbonne Universites, UPMC, Universite Paris 06, Ecole Normale Superiore, F-75005 \\ Paris, France \\ ${ }^{7}$ Centre for Astrophysics and Planetary Science, University of Kent, Canterbury, CT2 7NH, UK \\ ${ }^{8}$ Institute of Astronomy, Madingley Road, Cambridge, CB3 OHA, UK \\ ${ }^{9}$ Kavli Institute of Cosmology Cambridge, Madingley Road, Cambridge, CB3 OHA, UK \\ ${ }^{10}$ Université Laval, Pavillon Alexandre-Vachon 1045, Avenue de la Medecine, Canada \\ ${ }^{11}$ INAF-Osservatorio Astrofisico di Catania, Via S. Sofia 78, I-95123 Catania, Italy \\ Received 2020 September 25; revised 2021 February 11; accepted 2021 February 15; published 2021 April 16
}

\begin{abstract}
Massive star formation occurs in the interior of giant molecular clouds and proceeds through many stages. In this work, we focus on massive young stellar objects (MYSOs) and ultracompact H II regions (UCH II), where the former are enshrouded in dense envelopes of dust and gas, the latter of which has begun dispersing. By selecting a complete sample of MYSOs and UCH II from the Red MSX Source (RMS) survey database, we combine Planck and IRAS data and build their spectral energy distributions. With these, we estimate the physical properties (dust temperatures, mass, luminosity) of the sample. Because the RMS database provides unique solar distances, it also allows the instantaneous star formation efficiency (SFE) to be investigated as a function of Galactocentric radius. We find that the SFE increases between 2 and $4.5 \mathrm{kpc}$, where it reaches a peak, likely in correspondence with the accumulation of molecular material at the end of the Galactic bar. It then stays approximately constant up to $9 \mathrm{kpc}$, after which it linearly declines, in agreement with predictions from extragalactic studies. This behavior suggests the presence of a significant amount of undetected molecular gas at $R_{\mathrm{G}}>8 \mathrm{kpc}$. Finally, we present diagnostic colors that can be used to identify sites of massive star formation.
\end{abstract}

Unified Astronomy Thesaurus concepts: H II regions (694); Compact H II region (286); Young stellar objects (1834); Protostars (1302)

\section{Introduction}

The initial stages of a massive star can be traced back to a giant molecular cloud (GMC), i.e., a cloud with mass ranging from $10^{5}-10^{6} M_{\odot}$ (e.g., Dame et al. 2001; Miville-Deschenes et al. 2017) and a linear size up to hundreds of parsecs. Within GMCs, very dense molecular cores $\left(n>10^{5} \mathrm{~cm}^{-3}\right.$; e.g., Giannetti et al. 2013) collapse and evolve first into massive young stellar objects (MYSOs), and later, when the OB star begins to ionize the surrounding material, into ultracompact $\mathrm{H}$ II regions (UCH II).

In this framework, characterizing clumps hosting massive star formation, an intermediate stage between GMCs and cores, is of primary importance: massive star formation is known to take place in cold $\left(T_{d}<25 \mathrm{~K}\right)$, massive $\left(M>100 M_{\odot}\right)$, luminous $\left(L>10^{3} L_{\odot}\right)$ environments, so constraining dust temperatures, luminosities, and masses of the clumps allows one to assess whether the clump under investigation is able to effectively form massive stars. In general, determining accurate masses and luminosities for the clumps is also crucial to assess their evolutionary stage on a mass-luminosity plot (e.g., Molinari et al. 2008).

In this work, we are going to use the combined Planck High Frequency Instrument (HFI; Lamarre et al. 2010; Tauber et al. 2010; Planck Collaboration et al. 2011a; Planck HFI Core Team et al. 2011) and IRAS (Neugebauer et al. 1984) data to investigate a complete sample of clumps harboring MYSOs and UCH II regions. This study has two primary objectives: (1) to derive the properties (dust temperature, luminosity, mass, surface density) of these clumps and therefore fully characterize this evolutionary stage, and (2) to make use of the estimated luminosities and masses to compute an instantaneous star formation efficiency (SFE) for the first $0.1 \mathrm{Myr}$ (the approximate age of UCH II regions). For (2), we aim to address the following question: at this fixed evolutionary stage, is there variation in the amount of gas relative to the star formation (modulo the spread caused by the variation in age?) And, what could cause variations in the efficiency with which gas is converted into stars: galactic dynamics (sheer, bars), star formation mechanisms (cloud-cloud collisions), or hidden gas (e.g., undetected molecular material)?

The combination of the spectral bands from Planck and IRAS is extremely effective in probing the spectral energy distribution (SED) of sources such as MYSOs and UCH II. The coldest dust component present in these objects typically thermalizes at temperatures of the order of 15-20 K, and this makes the $850 \mu \mathrm{m}$ Planck band a very accurate tool in estimating the total mass. The accuracy of the Planck mass measurements is also due to the fact that this experiment operated from space, which means that no filtering for atmospheric noise is applied at any stage of data processing, 
hence emission on all angular scales is preserved. This is not the case for ground experiments such as APEX/LABOCA, JCMT/SCUBA, and CSO/Bolocam, where considerable flux loss occurs, with effects on the mass determination. An attempt to combine Planck and APEX/LABOCA data was carried out for the APEX Telescope Large Area Survey of the Galaxy (ATLASGAL; Schuller et al. 2009), a large and sensitive submillimeter survey of the inner Galactic plane. This work (Csengeri et al. 2016) though was limited to the investigation of the large-scale structure of cold dust.

Noteworthy, Planck and IRAS both mapped the whole sky, including both the inner and outer Galactic plane. The outer Galactic disk $\left(R_{\mathrm{G}}>8.5 \mathrm{kpc}\right)$ has characteristics that set it apart from the inner Galaxy. For instance, for $R_{\mathrm{G}}>13 \mathrm{kpc}$, both the $\mathrm{H}_{2}$ surface density (Scoville \& Sanders 1987; Digel et al. 1996; Heyer et al. 1998) and the stellar disk (Robin et al. 1992; Ruphy et al. 1996) appear to exhibit a sharp decline. In addition, the metallicity, at these large Galactocentric distances, is only half solar $\left(\sim 0.5 Z_{\text {sol }}\right.$; Yong et al. 2005). These observational facts suggest a dramatic change in star formation activity with respect to the inner Galaxy.

We emphasize that the Planck and IRAS beams (of the order of $5^{\prime}$ ) are sensitive to structures of $\sim 1.5$ pc (at a distance of $1.5 \mathrm{kpc}$ ), which is the typical size of Galactic clumps. Although clumps will contain multiple cores, we expect MYSOs or UCH II regions to be the dominant component within each individual clump.

The paper is organized as follows. Section 2 describes the Planck and the IRAS data sets. Section 3 provides details on the sample selection and on the identification of sources in Planck data. Section 4 discusses the photometric measurements, the SEDs, as well as the estimate of the clump dust temperatures, luminosities, masses, and surface densities. Section 5 presents the SFE in terms of clump luminosity-tomass ratio and its variations across the Galaxy. A summary is provided in the Conclusions (Section 6). Finally, the Appendix contains an estimate of the colors that can be used as diagnostics to identify regions of massive star formation.

\section{Planck Data}

Planck $^{12}$ (Tauber et al. 2010; Planck Collaboration et al. 2011a) was the third-generation space mission to measure the anisotropy of the cosmic microwave background (CMB). It observed the sky in nine frequency bands covering 30-857 $\mathrm{GHz}$ (i.e., 10,000-350 $\mu \mathrm{m}$ ), with high sensitivity and angular resolution from $31^{\prime}$ to $5^{\prime}$. The Low Frequency Instrument (LFI; Mandolesi et al. 2010) covered the 30, 44, and $70 \mathrm{GHz}$ bands with amplifiers cooled to $20 \mathrm{~K}$. The HFI (Lamarre et al. 2010; Planck HFI Core Team et al. 2011) covered the 100, 143, 217 , 353,545 , and $857 \mathrm{GHz}$ bands with bolometers cooled to $0.1 \mathrm{~K}$. Polarization was measured in all but the two highest frequency bands (Leahy et al. 2010; Rosset et al. 2010). Planck's sensitivity, angular resolution, and frequency coverage made it a powerful instrument for Galactic and extragalactic astrophysics as well as cosmology.

\footnotetext{
12 Planck (http://www.esa.int/Planck) is a project of the European Space Agency (ESA) with instruments provided by two scientific consortia funded by ESA member states (in particular the lead countries France and Italy), with contributions from NASA (USA) and telescope reflectors provided by a collaboration between ESA and a scientific consortium led and funded by Denmark.
}

In this paper, we use the Planck PR2 full-channel, fullmission temperature maps at nominal frequencies 353 $(850 \mu \mathrm{m}), 535(560 \mu \mathrm{m})$, and $857 \mathrm{GHz}(350 \mu \mathrm{m})$. We downloaded the maps from the IRSA archive. ${ }^{13}$ The $353 \mathrm{GHz}$ map are in $T_{\mathrm{CMB}}$ units, while the 535 and $857 \mathrm{GHz}$ maps are in MJy $\mathrm{sr}^{-1}$. To perform the conversion from CMB thermodynamic units to Rayleigh-Jeans brightness temperature units, we make use of the conversion factor given in Table 3 of Planck Collaboration et al. (2016a).

We adopt the effective beam sizes provided in the Explanatory Supplement. ${ }^{14}$ These are 4!86, 4!84, and 4!.63 at, respectively, 353, 545, and $857 \mathrm{GHz}$.

\subsection{Ancillary Data}

In order to construct the SEDs of the clumps, we complement the Planck data with IRAS (Neugebauer et al. 1984) data at 100, 60, and $25 \mu \mathrm{m}$. In particular, we use the IRIS (Improved Reprocessing of the IRAS Survey; MivilleDeschenes \& Lagasche 2005) maps, which benefit from the COBE-DIRBE (Hauser et al. 1998) calibration and zero point, as well from a better zodiacal light subtraction and destriping. We do not include the IRIS $12 \mu \mathrm{m}$ data because this band is significantly contributed to by emission from polycyclic aromatic hydrocarbons (PAHs), whose modeling and characterization are beyond the scope of this paper. The FWHM of the IRIS beams are, respectively, $3 ! 8,4 ! 0$, and $4 ! 3$, at 25,60 , and $100 \mu \mathrm{m}$.

Both the Planck and IRIS data are reprojected into the HEALPix (Hierarchical Equal Area isoLatitude Pixelization; Gorski et al. 2005) format with Nside $=2048$. All maps are smoothed to the Planck $353 \mathrm{GHz}$ angular resolution (4!86).

\section{Sample Selection}

Our reference database for the source selection is the Red MSX Source (RMS) ${ }^{15}$ survey (Lumsden et al. 2013), i.e., a campaign of follow-up observations designed to identify possible contaminants (e.g., evolved stars and planetary nebulae, PNe) of a sample of $\sim 2000$ candidate MYSOs assembled by Lumsden et al. (2002) using MSX color selection criteria. Examples of the follow-up observations that have been undertaken by the RMS team are high-resolution ( $\left(1^{\prime \prime}\right)$ radio continuum observations (Urquhart et al. 2007a, 2009) that have allowed the discrimination of UCH II regions from $\mathrm{PNe}$ and near-IR spectroscopic measurements that have led to the identification of evolved stars (Clarke et al. 2006). This effort has made it possible to single out some 1500 (1420) MYSOs and UCH II regions with uniquely constrained distances (Urquhart et al. 2013). Radial velocities were obtained from the ${ }^{13} \mathrm{CO} J=1-0$ and $J=2-1$ observations (Urquhart et al. 2007b, 2008), and these have been coupled with the Reid et al. (2009) rotation curve to derive kinematic distances.

See Figure 1 for examples of MYSOs and UCH II regions from the RMS database in the IRAS/IRIS and Planck data.

Mottram et al. (2011) investigated the completeness of the RMS sample. To this end, they computed the volume of the Galaxy probed by the RMS survey at each luminosity $L$ and assumed that the RMS MYSOs and H II regions are distributed

\footnotetext{
${ }^{13}$ https://irsa.ipac.caltech.edu/data/Planck/release_2/

14 https://wiki.cosmos.esa.int/planck-legacy-archive/index.php/Effective_ Beams

15 http://www.ast.leeds.ac.uk/RMS/
} 

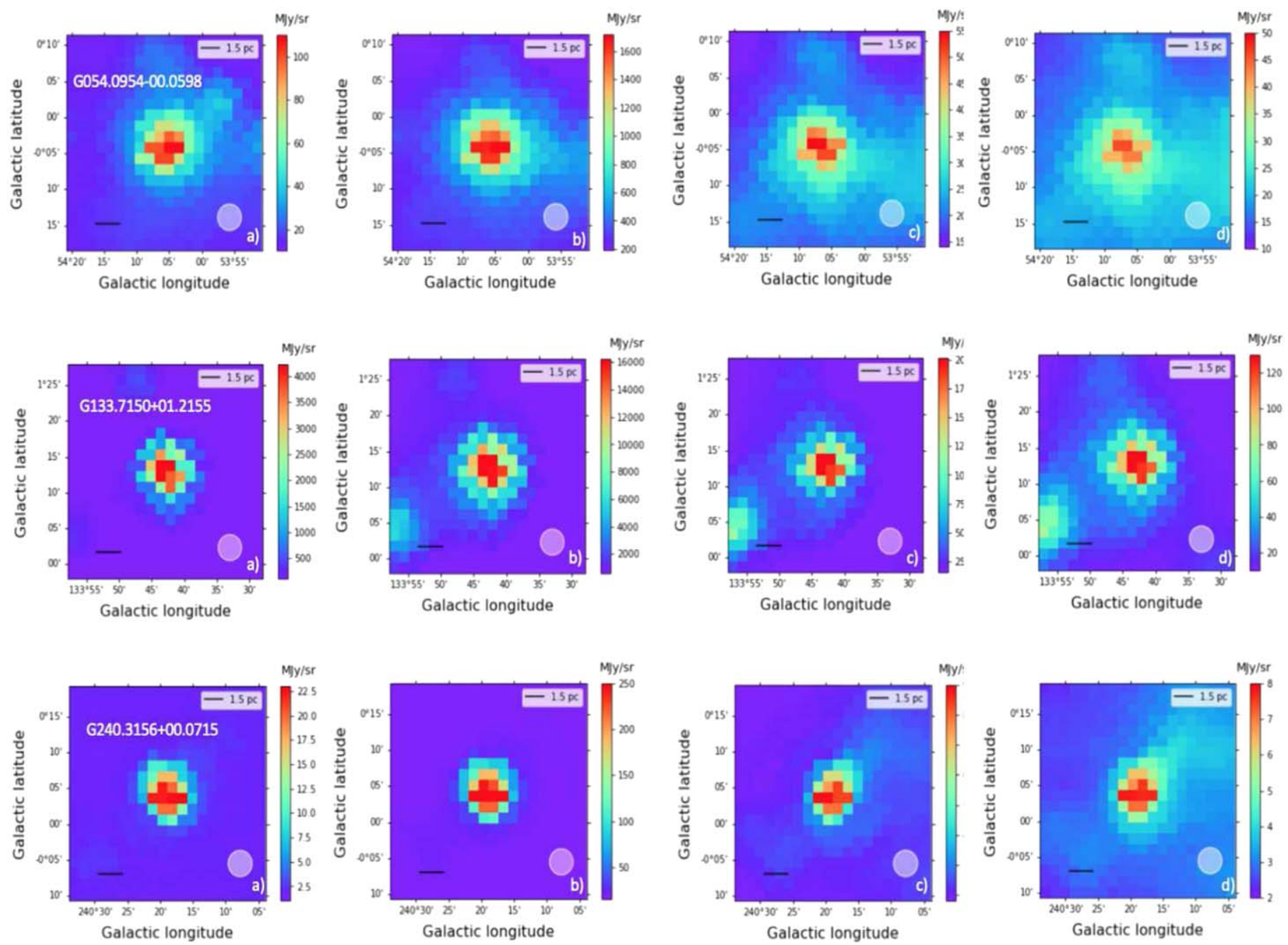

Figure 1. Examples of MYSOs and UCH II regions identified in the Planck data at the RMS position. From left to right: (a) IRIS $25 \mu \mathrm{m}$, (b) IRIS $100 \mu \mathrm{m}$, (c) Planck HFI $857 \mathrm{GHz}(350 \mu \mathrm{m})$, and (d) Planck HFI $353 \mathrm{GHz}(850 \mu \mathrm{m})$. All of the data are convolved to the common Planck HFI $353 \mathrm{GHz}$ angular resolution $(4$. 86 ).
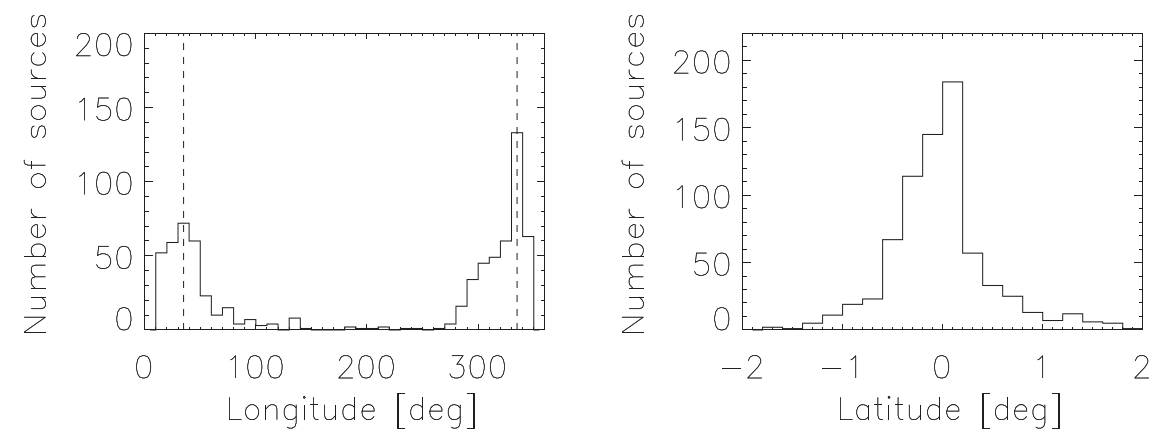

Figure 2. Longitude (left panel) and latitude (right panel) distributions of the sample of candidate MYSOs and UCH II regions above the $10^{4} L_{\odot}$ completeness limit. The dashed lines on the longitude plot denote, respectively, the Sagittarius and Scutum-Crux arms (at $\left.l \sim 30^{\circ}\right)$ and the Norma and Scutum-Crux arms $\left(\right.$ at $l \sim 330^{\circ}$ ).

in a layer with a hole in the center, according to the model by Robin et al. (2003) for the thin-disk Galactic stellar population. Following this method, they obtained that the survey is $100 \%$ complete for luminosities greater than $\sim 1 \times 10^{4} L_{\odot}$. This selection leads to 731 sources.

The longitude and latitude distributions of the complete sample are shown in Figure 2. From the longitude distribution, it is evident that there is a higher source concentration toward the intersection of the line of sight with the Sagittarius and Scutum-Crux arms (first Galactic quadrant, $l \sim 30^{\circ}$ ) and with the Norma and Scutum-Crux arms (fourth Galactic quadrant, $l \sim 330^{\circ}$ ). Out of 731 sources, 209 are MYSO candidates, 509 are UCH II regions, and 13 are thought to be transition objects, i.e., sources that are older than MYSOs but not old enough to be classified as UCH II regions. In addition, $151(\sim 20 \%)$ are located at Galactocentric distances greater than $8.5 \mathrm{kpc}$, and 108 are part of complexes of multiple sources.

For each source in the RMS data, we create a $30^{\prime} \times 30^{\prime}$ image centered on the source at each Planck frequency (353, $545,857 \mathrm{GHz}$ ). Each image is visually inspected to ensure that 
the emission in the considered region is not dominated by background emission. From this visual inspection, we notice that strong sources of emission centered on the location of the RMS source are, with a few exceptions $(\sim 5 \%)$, present in the Planck HFI bands.

To estimate the angular size of these structures, we follow two complementary approaches. First, we assume that all of the sources are unresolved with respect to the Planck $353 \mathrm{GHz}$ convolution beam $(4 ! 86)$; second, we perform a two-dimensional Gaussian fit and, for each clump, we derive a measure of the major and minor axes at $353 \mathrm{GHz}(850 \mu \mathrm{m})$. In this case, not all sources turn out to be resolved. Out of 731, only for 238 $(32.5 \%)$ can we determine the major and minor axes, with an average size of $5.6 \pm 1.5$. In the following sections, we will only show the results for the unresolved case, while we will annotate in parentheses the results for the fitted size case.

\section{SEDs}

To compute the flux in each band, we use the HEALpix aperture photometry code developed for Planck Collaboration et al. (2011c). As input aperture radius, we use half the convolution beam FWHM (4!86) if the sources are unresolved; otherwise, we use half of the estimated FWHM from the 2D Gaussian fit. In case the sources are quasi-spherically symmetric, i.e., their aspect ratio is $\sim 1$, the major and minor axes are averaged together and half this average is used as the source aperture, aper $_{s}$. Alternatively, half of the major axis is used as the aperture.

After converting the maps in units of Jy/pixel, the pixels within an aperture equal to $\operatorname{aper}_{s}$ are summed together. An estimate of the background is subtracted using a median estimator of the pixels within radii $\left[\operatorname{aper}_{s}, 2 \times\right.$ aper $_{s}$ ]. Uncertainties are obtained by summing in quadrature the rms of the values in the background annulus to the absolute calibration uncertainties for each map.

For the sources in double (90) or triple systems (18), in order to avoid double-counting, we have subdivided the total flux in the Planck beam by two or three, depending on the case.

Since the pioneering works of Chini et al. (1986a, 1986b, 1986c, 1987), it has been known that, in order to explain observations of $\mathrm{H}$ II regions above and below $\sim 100 \mu \mathrm{m}$, one has to invoke the existence of a two-temperature component dust distribution: a warm, low-density population of dust grains situated in the proximity of the central source, and a colder dust population in the periphery of the cloud. The preliminary finding by Chini et al. was subsequently confirmed by data at increasingly higher spatial resolution and larger spectral coverage (e.g., Povich et al. 2007), as well as by sophisticated radiative transfer modeling (e.g., Zhang \& Tan 2011). The SEDs obtained from the combination of the Planck and IRIS data show a behavior similar to the one just described (Figure 3, blue points), as the IRIS $60 \mu \mathrm{m}$ and $25 \mu \mathrm{m}$ data points cannot be represented by the same graybody as the measurements at longer wavelengths. Taking this fact into account, we fit our SEDs with the functional form

$$
S_{\lambda}=A_{1}\left(\frac{\lambda}{\lambda_{0}}\right)^{-\beta} B_{\lambda}\left(T_{c}\right)+A_{2}\left(\frac{\lambda}{\lambda_{0}}\right)^{-\beta} B_{\lambda}\left(T_{w}\right),
$$

where $T_{c}$ and $T_{w}$ are, respectively, the temperatures of the cold and warm components, and $\lambda_{0}$ is set to $100 \mu \mathrm{m}$.
To estimate the parameters $A_{1}, A_{2}, T_{c}$, and $T_{w}$, we use a $\chi^{2}$ goodness-of-fit method. For the spectral emissivity index, $\beta$, we test three different values: $1.8,2.0$, and 2.2. The best $\chi^{2}$ are consistently given by fits performed with $\beta=1.8$ (see Table 1); therefore, this is the value that we adopt for both graybodies in the final runs.

Note that we do not perform the photometric SED fitting by using a more sophisticated modeling (e.g., Robitaille et al. 2006, 2007) for several reasons: at the angular resolution we are working, we are not sensitive to the parameters probed by these models (e.g., disk inner/outer radius, cavity opening angle, etc.); moreover, we are probing the extent of clumps, while those frameworks were designed for describing the behavior of individual cores; finally, UCH II represent a far too advanced evolutionary stage not accounted for by those models.

An illustration of the result of the fits is provided in Figure 3 (red solid line). Occasionally, in one (or more) HFI frequency band, the best-fit model slightly over-/underpredicts the measured data points. This is a consequence of the adopted value for $\beta$. We have tested this hypothesis by making trial fits with the spectral emissivity index as a free parameter. Although in some cases the fit $\chi^{2}$ improves, we prefer to keep $\beta$ fixed and equal to the canonical value of 1.8 , as the analysis of variations of $\beta$ and of its degeneracy with respect to the dust temperature (e.g., Schnee et al. 2007; Juvela et al. 2013, 2018) is beyond the scope of this paper.

\subsection{Dust Temperature Distribution}

From the fitting procedure, we derive estimates for the cold $\left(T_{c}\right)$ and warm $\left(T_{w}\right)$ dust temperatures components, $\overline{T_{c}}=21.2 \pm 2.9 \mathrm{~K}(21.2 \pm 3.1 \mathrm{~K}$ for the fitted size case $)$ and $\overline{T_{w}}=54.0 \pm 4.6 \mathrm{~K}(53.8 \pm 4.4 \mathrm{~K}$ for the fitted size case $)$, i.e., comparable to those found for more evolved H II regions (e.g., Povich et al. 2007; Paladini et al. 2012). For the uncertainties, rather than the modeling errors, we adopt the standard deviations of the sample, given that the former underestimates the true errors. König et al. (2017) investigate a small sample of MYSOs (36 sources) and UCH II regions (25 sources), and for these, they derive dust temperatures from the modeling of the combined MSX (Egan et al. 2003), WISE (Wright et al. 2010), Hi-GAL, and ATLASGAL data. They obtain mean temperatures of $28.1 \pm 3.6 \mathrm{~K}$ and $31.7 \pm 4.0 \mathrm{~K}$ for MYSOs and UCH II regions, respectively, which lie in between our average cold and warm components. This work was extended to the whole ATLASGAL sample by Urquhart et al. (2018), who find, for MYSOs and H II regions, values of dust temperature between $\sim 15$ and $40 \mathrm{~K}$.

We subdivide the sample into six Galactocentric bins of equal width (i.e., $2 \mathrm{kpc}$ ) and for each bin, we compute the average $T_{c}$ and $T_{w}$ (see Table 2). The distributions of $T_{c}$ and $T_{w}$ versus $R_{\mathrm{G}}$ are plotted in Figure 4 (top panel) and indicate decreasing temperature for the cold component toward the outer Galaxy, in particular for $R_{\mathrm{G}}>10 \mathrm{kpc}$, accompanied by an opposite trend for the warm component.

An important caveat to keep in mind is that, by binning according to distance from the Galactic center, we assume that the Galaxy is radially symmetric and, in doing so, we ignore the presence of spiral arms. To take this effect into account and explore even further dust temperature variations across the Galaxy, we split the sample according to longitude. We denote as inner-Galaxy sources those located either in the 

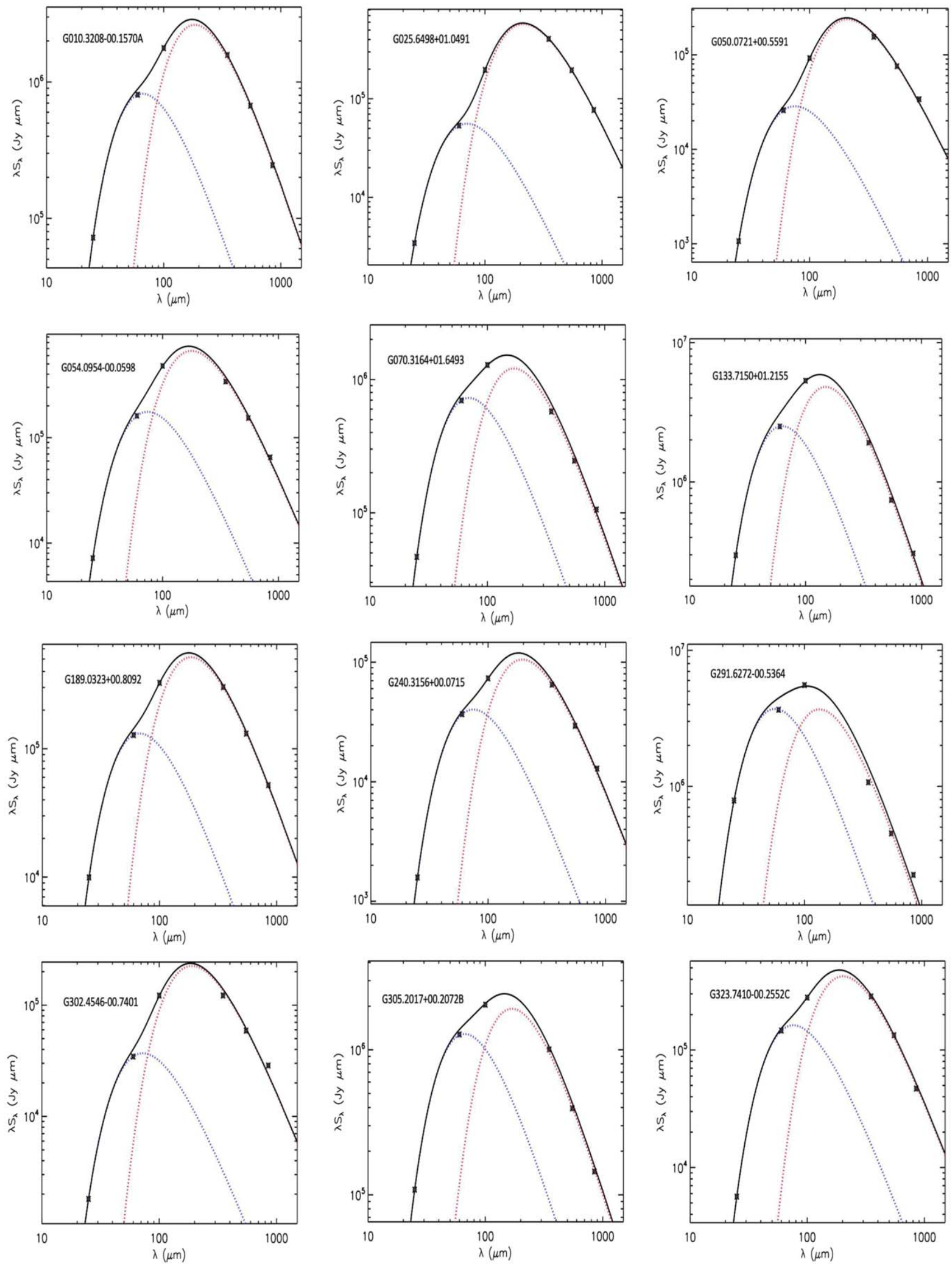

Figure 3. Examples SED fits to the Planck and IRIS clumps fluxes. The best-fit two-temperature component model is shown (black solid line). Black diamonds denote the Planck 350, 500, and $850 \mu \mathrm{m}$ and IRIS 100, 60, and $25 \mu \mathrm{m}$ data points. The cold (blue dashed line) and warm (red dashed line) temperature components are also shown. 
Table 1

Spectral Emissivity Index, $\beta$, and Corresponding Average SED Fitting $\chi^{2}$

\begin{tabular}{lc}
\hline \hline$\beta$ & $\chi^{2}$ \\
\hline 1.8 & 7.3 \\
2.0 & 15.9 \\
2.2 & 31.2 \\
\hline
\end{tabular}

first $\left(0^{\circ}<l<90^{\circ}\right)$ or fourth $\left(270^{\circ}<l<360^{\circ}\right)$ Galactic quadrants, and as outer-Galaxy sources those either in the second or third Galactic quadrants $\left(90^{\circ}<l<270^{\circ}\right)$. We have 699 candidate MYSOs/UCH II regions in the inner sample and 32 in the outer one. Then, for each dust temperature component ( $T_{c}$ or $T_{w}$ ), we compare the histogram distribution for the inner-/outer-Galaxy subsamples (Figure 4, middle panels). In this case, the average temperatures are $\overline{T_{c, i}}=21.2 \pm 2.9 \mathrm{~K}$ $\left(21.2 \pm 3.1 \mathrm{~K}\right.$ for the fitted size case), $\overline{T_{c, o}}=20.8 \pm 2.4 \mathrm{~K}$ $(20.6 \pm 2.7 \mathrm{~K}), \overline{T_{w, i}}=53.7 \pm 4.4 \mathrm{~K}(53.5 \pm 4.3 \mathrm{~K}$ for the fitted size case), and $\overline{T_{w, o}}=59.5 \pm 4.6 \mathrm{~K}(58.8 \pm 4.6 \mathrm{~K}$ for the fitted size case), where $T_{c, i} / T_{c, o}$ and $T_{w, i} / T_{w, o}$ are the cold/ warm dust temperatures for the inner-/outer-Galaxy subsamples, respectively. The histogram distributions highlight a trend similar to what is found in Figure 4 (upper panels).

We interpret this result in the context of the large-scale properties of the Galactic plane. A dust temperature gradient is known to characterize the diffuse emission along the Galactic plane, with temperatures ranging from $14-15 \mathrm{~K}$ in the outer plane, to $\simeq 19 \mathrm{~K}$ for the inner one (Planck Collaboration et al. 2011b). The temperature enhancement toward the center of the Galaxy is typically explained as due to the presence of a high concentration of star-forming regions, especially in correspondence with the molecular ring $\left(R_{\mathrm{G}} \sim 5 \mathrm{kpc}\right)$. From our analysis, it appears that the small-scale behavior reflects the situation on larger scales, with clumps harboring massive stars at large Galactic radii being at lower temperatures with respect to their counterparts closer to the Galactic center. Because $T_{D} \propto X_{\mathrm{ISRF}}^{1 /(4+\beta)}$ —with $T_{D}$ denoting the dust temperature, XISRF the intensity of the interstellar radiation field (ISRF), and $\beta$ the dust emissivity index, if we assume a Mathis et al. (1983) radiation field, i.e., scaling with the inverse of $R_{G}$, we obtain that the clumps' cold dust component appears to be a local measure of the global radiation field. On the contrary, the clumps' warm dust component depends on the stellar radiation field, since this dust is located in the proximity of the young massive stars. Remarkably, an O5 star will heat up dust up to $30 \mathrm{~K}$ out to a radius of $0.75 \mathrm{pc}$ from the star (Whitney et al. 2005). The trend that we find appears to mimic the well-known inversely proportional increase of electron temperature, $T_{e}$, with galactocentric radius in $\mathrm{H}$ II regions (Shaver et al. 1983, Paladini et al. 2004), which is a consequence of the Galactic metallicity gradient and of the fact that metals such as oxygen are coolants.

A dependence of dust temperature on Galactocentric distance is also reported by Urquhart et al. (2018). In their analysis, these authors do not distinguish between cold and warm temperature components; however, they find increasing temperatures at larger Galactocentric distances.

Finally, we have analyzed potential differences between the warm/cold dust temperatures of candidate MYSOs and UCH II regions (Figure 4, bottom panel). We do not find any indication that these two populations are characterized by different temperatures. We find $\overline{T_{c, \mathrm{MYSO}}}=20.5 \pm 3.0 \mathrm{~K}$ (same for the fitted size case), $\overline{T_{w, \text { MYSO }}}=54.5 \pm 5.0 \mathrm{~K}(54.3 \pm 4.9 \mathrm{~K}$ for the fitted size case), $\overline{T_{c, \mathrm{UCH}_{\mathrm{II}}}}=21.5 \pm 2.8 \mathrm{~K}(21.5 \pm 3.0 \mathrm{~K}$ for the fitted size case), and $\overline{T_{w, \mathrm{UCH}_{\mathrm{II}}}}=53.8 \pm 4.4 \mathrm{~K}(53.6 \pm$ $4.2 \mathrm{~K}$ for the fitted size case), where $T_{c, \mathrm{MYSO}} / T_{c, \mathrm{UCH}_{\mathrm{II}}}$ and $T_{w, \mathrm{MYSO}} / T_{w, \mathrm{UCH}_{\mathrm{II}}}$ are the cold/warm dust temperatures for the MYSOs/UCH II regions.

\subsection{Luminosities, Masses, and Surface Densities}

Having fitted the source SEDs, we can compute their luminosities. To this end, we use the relation

$$
L_{\mathrm{IR}}=4 \pi D^{2} \int_{\lambda_{\min }}^{\lambda_{\max }} S_{\lambda} d \lambda,
$$

where $S_{\lambda}$ is the best-fit two-temperature component model discussed in Section 4 , and $D$ is the solar distance from the RMS database. The integration is performed between $25 \mu \mathrm{m}$ and $850 \mu \mathrm{m}$, i.e., the shortest/longest IRIS/Planck wavelength considered in our analysis. The result is shown in Figure 5. We obtain $\overline{L_{\mathrm{IR}}}=8.3 \times 10_{-6.5 \times 10^{4}}^{5+1.4 \times 10^{6}} L_{\odot}\left(\bar{L}=1.1 \times 10_{-6.8 \times 10^{4}}^{6+1.7 \times 10^{6}} L_{\odot}\right.$ for the fitted size case). We find that sources in the inner Galaxy are on average more luminous than in the outer Galaxy, with $\overline{L_{\mathrm{IR} \text {,inner }}}=8.9 \times 10_{-6.9 \times 10^{4}}^{5+1.5 \times 10^{6}} L_{\odot}$ $\left(\overline{L_{\mathrm{IR}, \text { inner }}}=1.2 \times 10_{-7.6 \times 10^{4}}^{6+1.8 \times 10^{6}} L_{\odot}\right.$ for the fitted size case $)$ and $\overline{L_{\mathrm{IR}, \text { outer }}}=3.0 \times 10_{-2.8 \times 10^{4}}^{5+6 \times 10^{5}} L_{\odot}\left(\overline{L_{\mathrm{IR}, \text { outer }}}=3.6 \times 10_{-3.1 \times 10^{4}}^{5+6.2 \times 10^{5}} L_{\odot}\right.$ for the fitted size case). We also look into differences between the average luminosity of clumps associated with MYSOs and UCHII regions and the latter turn out to be more qluminous, with $\overline{L_{\mathrm{IR}, \mathrm{UCHII}}}=9.6 \times 10_{-7.7 \times 10^{4}}^{5+1.6 \times 10^{6}} L_{\odot}$ $\left(\overline{L_{\mathrm{IR}, \mathrm{UCHII}}}=1.3 \times 10_{-8.2 \times 10^{4}}^{61.9 \times 10^{6}} L_{\odot}\right.$ for the fitted size case $)$ and $\overline{L_{\mathrm{IR}, \mathrm{MYSO}}}=5.3 \times 10_{-4.3 \times 10^{4}}^{5+7.8 \times 10^{5}} L_{\odot}\left(\overline{L_{\mathrm{IR}, \mathrm{MYSO}}}=6.7 \times 10_{-4.4 \times 10^{4}}^{5+8.7 \times 10^{5}} L_{\odot}\right.$ for the fitted-size case), which is consistent with their more advanced evolutionary stage. This is also similar to what has been reported by Urquhart et al. (2014), who analyzed a sample of $~ 800$ ATLASGAL clumps associated with $\sim 1000$ RMS sources. These authors compute their luminosities by using the model SED fitter developed by Robitaille et al. (2007) and by combining flux measurements from 2MASS (Skrutskie et al. 2006), UKIDSS (Lucas et al. 2008), or Vista-VVV (Minniti et al. 2010), MSX, WISE, Hi-Gal, and ATLASGAL. They obtain that the luminosity distributions of the MYSO and UCH II regions subsamples are significantly different, with the two distributions peaking at $\sim 1 \times 10^{4} L_{\odot}$ and $\sim 4 \times 10^{4} L_{\odot}$, respectively. This difference in the luminosity function was first discussed in Mottram et al. (2011). Note that the lower luminosities reported by Urquhart et al. (2014) with respect to our values are likely due to the fact that the ATLASGAL clumps are smaller in size, i.e., on average $\sim 1.2$ pc (see below for a comparison with our average size).

For the calculation of clump masses, assuming thermal equilibrium, we have:

$$
M_{\mathrm{IR}}=\frac{S_{850 \mu \mathrm{m}} D^{2}}{\kappa_{850 \mu \mathrm{m}} B_{\nu}\left(T_{d}\right)}
$$

where the dust opacity, assuming a gas-to-dust ratio of 100 , $\kappa_{850 \mu \mathrm{m}}=\kappa_{353 \mathrm{GHz}}=0.012 \mathrm{~cm}^{2} \mathrm{~g}^{-1}$ (Preibisch et al. 1993), and $T_{d}=T_{c}$. Given that we only consider the cold dust 
Table 2.

Average Cold $\left(T_{c}\right)$ and Warm $\left(T_{w}\right)$ Dust Temperatures per Galactocentric Bin for the Two Cases of Unresolved Sources and Fitted Diameters

\begin{tabular}{lcccc}
\hline \hline $\begin{array}{l}\text { Bin Center } \\
(\mathrm{kpc})\end{array}$ & No. Sources & $\begin{array}{c}T_{c} \text { (Unresolved) } \\
(\mathrm{K})\end{array}$ & $\begin{array}{c}T_{w} \text { (Unresolved) } \\
(\mathrm{K})\end{array}$ & $\begin{array}{c}T_{c} \text { (Fitted Sizes) } \\
(\mathrm{K})\end{array}$ \\
\hline 3 & & $21.3 \pm 1.6$ & $51.5 \pm 2.9$ & $21.2 \pm 1.5$ \\
5 & 57 & $21.5 \pm 2.9$ & $53.2 \pm 4.6$ & $21.6 \pm 3.2$ \\
$(\mathrm{~K})$
\end{tabular}

component and ignore dust depletion effects in the source proximity, our computed mass will be an overestimate of the true values. Also, we emphasize that changing the value of the adopted dust opacity will affect the mass estimates. For instance, Ossenkopf \& Henning (1994) give $\kappa_{700 \mu \mathrm{m}}=$ $0.0257 \mathrm{~cm}^{2} \mathrm{~g}^{-1}$, causing masses to be a factor of 2 lower. Finally, the dust-to-gas ratio, rather than being a fixed value across the Galaxy, varies with Galactocentric distance, and this will introduce further uncertainties into the mass determinations.

Figure 6 illustrates the overall mass distribution (top panel), the inner and outer Galaxy mass distribution (middle panel), and the mass distribution for MYSOs, UCH II regions, and MYSOs/UCH II. The resulting average mass is $\overline{M_{\mathrm{IR}}}=7.7 \times$ $10_{-0.9 \times 10^{3}}^{4+1.3 \times 10^{4}} M_{\odot}\left(\overline{M_{\mathrm{IR}}}=1.1 \times 10_{-1.0 \times 10^{3}}^{4+1.8 \times 10^{4}} M_{\odot}\right.$ for the fitted size case). The sources in the inner Galaxy are not only more luminous but also more massive compared to those in the outer Galaxy; in fact, $\overline{M_{\mathrm{IR}, \text { inner }}}=8.2 \times 10_{-1.15 \times 10^{3}}^{3+1.4 \times 10^{4}} M_{\odot}$ $\left(\overline{M_{\mathrm{IR}, \text { inner }}}=1.2 \times 10_{-1.2 \times 10^{3}}^{4+1.8 \times 10^{4}} M_{\odot}\right.$ for the fitted size case) and $\overline{M_{\mathrm{IR}, \text { outer }}}=2.2 \times 10_{-0.4 \times 10^{3}}^{3+4.5 \times 10^{3}} M_{\odot}\left(\overline{M_{\mathrm{IR}, \text { outer }}}=3.3 \times 10_{-0.6 \times 10^{3}}^{3+5.3 \times 10^{3}} M_{\odot}\right.$ for the fitted size cases). Finally, clumps associated with UCH II regions are more massive than clumps associated with MYSOs, with $\overline{M_{\mathrm{IR}, \mathrm{UCHII}}}=8.5 \times 10_{-1.1 \times 10^{3}}^{3+1.4 \times 10^{4}} M_{\odot} \quad\left(\overline{M_{\mathrm{IR}, \mathrm{UCHII}}}=1.2 \times\right.$ $10_{-1.1 \times 10^{3}}^{4+1.9 \times 10^{4}} M_{\odot}$ for the fitted size case) and $\overline{M_{\mathrm{IR}, \mathrm{MYSO}}}=5.5 \times$ $10_{-0.7 \times 10^{3}}^{3+9.9 \times 10^{3}} M_{\odot} \quad\left(\overline{M_{\mathrm{IR}, \mathrm{MYSO}}}=6.8 \times 10_{-0.9 \times 10^{3}}^{3+1.2 \times 10^{4}} M_{\odot} \quad\right.$ for the fitted size case), again in agreement with the former being older objects that have completely undergone the accretion process. A similar result is reported in Urquhart et al. (2014) based on the analysis of the sample of ATLASGAL clumps (see above). They find that the MYSO and UCH II regions associated with clump distributions have a median value of $\sim 1000 M_{\odot}$ and $2800 M_{\odot}$, respectively. Note, once again, that the lower ATLASGAL values can be ascribed to the smaller clump sizes.

In the overall sample, we have identified a subsample of 15 very massive objects, i.e., with mass $>4 \times 10^{4} M_{\odot}$. These are 3 MYSOs and $12 \mathrm{UCH}$ II regions, which are concentrated toward $l \sim 43^{\circ}$ and $l \sim 338^{\circ}$, at a Galactocentric distance of, respectively, $7 \mathrm{kpc}$ and $5 \mathrm{kpc}$.

The derived masses allow us to estimate the source's surface densities, $\Sigma=M_{\mathrm{IR}} /\left(\pi \mathrm{r}^{2}\right)$, where $r$ is the linear radius. We obtain a mean surface density equal to $0.25_{-0.003}^{+0.17} \mathrm{~g} \mathrm{~cm}^{-2}$ (Figure 7). This mean value is greater than the characteristic surface densities of GMCs, $\Sigma \sim 0.035 \mathrm{~g} \mathrm{~cm}^{-2}$ (Solomon et al. 1987), indicating that the clumps in our sample are gravitationally bound (Bertoldi \& McKee 1992; Williams et al. 2000). At the same time, this is lower than the average surface density (i.e., $\Sigma \geq 1 \mathrm{~g} \mathrm{~cm}^{-2}$ ) found by Plume et al. (1997) for a sample of regions of massive star formation observed in the $J=5 \rightarrow 4$ and $2 \rightarrow 1$ transitions of CS and $C^{34} S$. Importantly, Plume's sources have a mean linear radius and (virial) mass of $0.5 \mathrm{pc}$ and $3800 M_{\odot}$, respectively. Our sample is characterized by generally larger and more massive clumps, with a mean linear radius of $4.7 \pm 3.7 \mathrm{pc}$, and they do not represent the dusty counterparts of the molecular dense clumps studied by Plume et al. (1997): while Plume et al.'s observations trace the densest material directly involved in the production of massive stars, the Planck measurements are sensitive to the total mass involved, including less dense material. More recent studies (Butler \& Tan 2012; Traficante et al. 2018, 2020) have revised down the massive star formation threshold value, and today, it is generally accepted that this is to be found in the range $0.1-0.35 \mathrm{~g} \mathrm{~cm}^{-2}$, in agreement with the fact that our clumps are forming massive stars.

\section{Variation of the Star Formation Efficiency with Galactocentric Radius}

It is well established that the outer Galaxy presents a very different environment with respect to the inner Galaxy, with the former characterized by low total gas densities, low metallicities, and gas abundances, as well as by higher shear. Because of the Kennicutt (1998) law, the low densities make the gas gravitationally stable, hence not apt to star formation. Despite these unfavorable environmental conditions, sporadic star formation is observed toward the outer Galaxy, suggesting that mechanisms other than gravity may trigger the star formation process. In particular, Elmegreen \& Hunter (2006) propose that some degree of turbulence persists in the outer disk, allowing the formation of clouds and compensating for the lack of gravitational instabilities.

A measure of the global instantaneous SFE can be obtained from the ratio of the IR luminosity, $L_{\mathrm{IR}}$, to clump mass, $M_{\mathrm{IR}}$. Both of these quantities have the same square dependence on distance $\left(D^{2}\right)$, therefore their ratio is distance independent. This is very important given that solar distances, especially when derived from kinematic measurements, can be affected by large uncertainties. Using the same binning in Galactocentric radii described in Section 4.1, we then investigate how the luminosity-to-mass ratio, $L_{\mathrm{IR}} / M_{\mathrm{IR}}$, varies from the inner to the outer Galaxy. For this purpose, we first analyze the $L_{\mathrm{IR}} / M_{\mathrm{IR}}$ distributions per Galactocentric bin (Figure 8). Then, we average the $L_{\mathrm{IR}} / M_{\mathrm{IR}}$ values in each bin, and look at the distribution of the mean values as a function of Galactocentric radius (Figure 9 and Table 3). It is apparent from Figure 8 that there is a significant dispersion of the $L_{\mathrm{IR}}$ to $M_{\mathrm{IR}}$ ratios in each bin, indicating that star formation does not simply scale with distance from the Galactic center, but instead is a complex 
The Astrophysical Journal, 911:69 (13pp), 2021 April 10
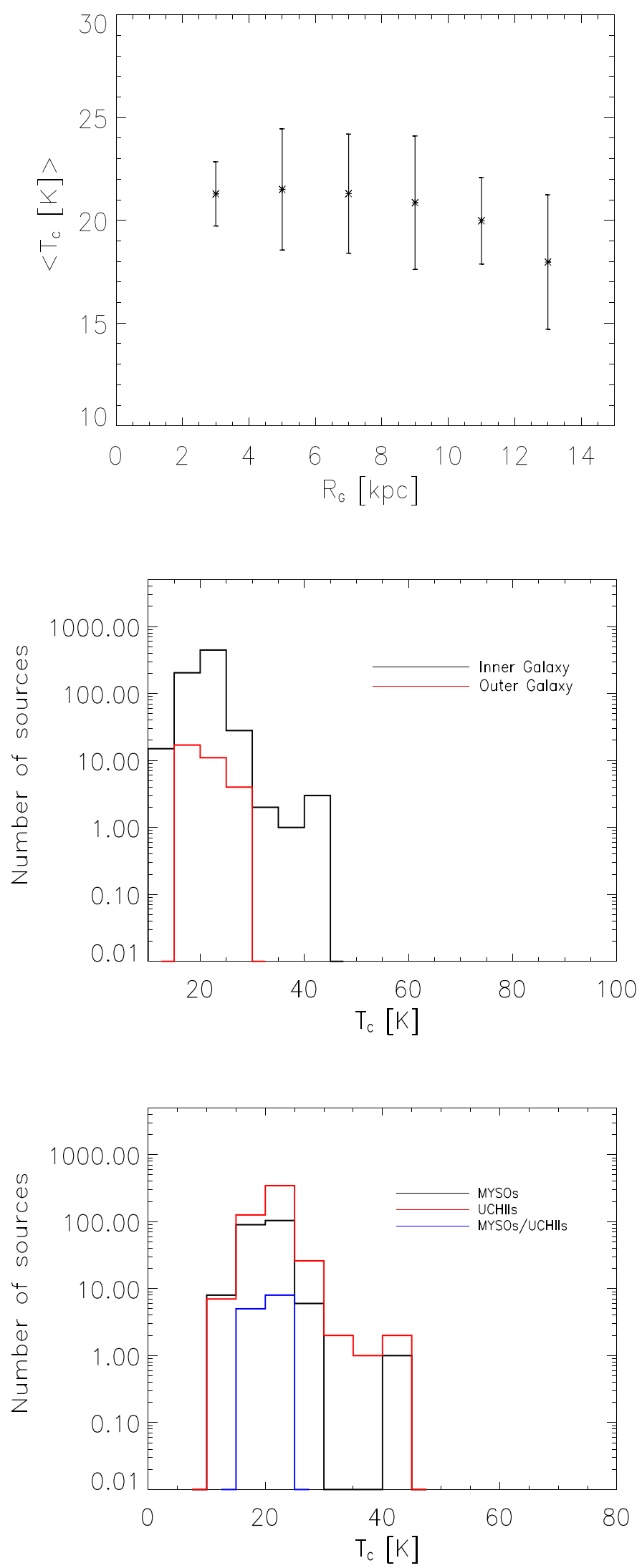

Paladini et al.

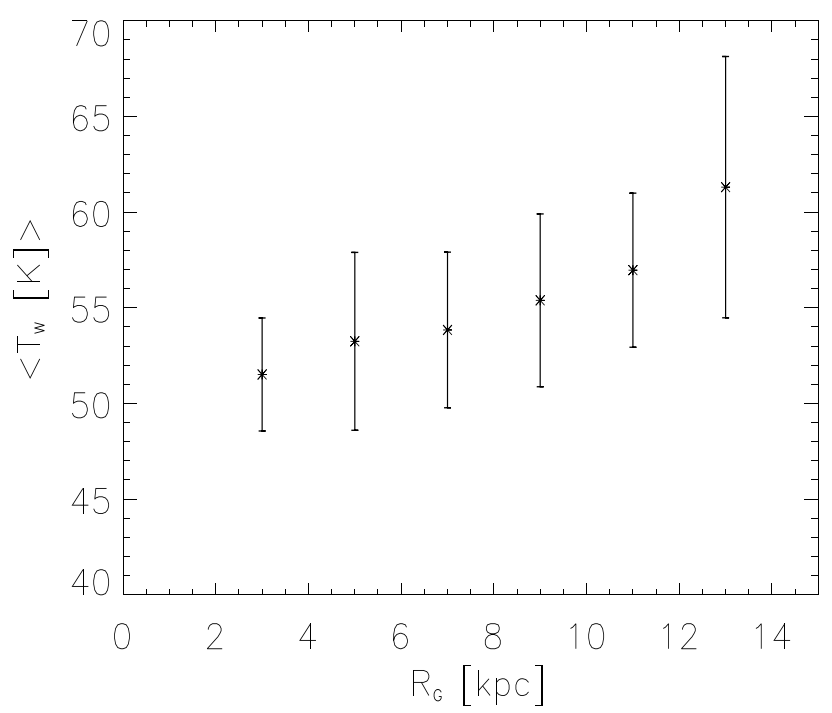

Figure 4. Top: average cold $\left(T_{c}\right)$ and warm $\left(T_{w}\right)$ dust temperature components as a function of Galactocentric radius. $R_{\mathrm{G}}$ values are sampled in $2 \mathrm{kpc}$ bins. The plotted error bars are the standard deviations in each bin. Middle: cold and warm dust temperature distributions for sources in the inner (first and fourth) and outer (second and third) Galactic quadrants. Bottom: cold and warm dust temperature distributions for MYSOs and UCH II regions. 


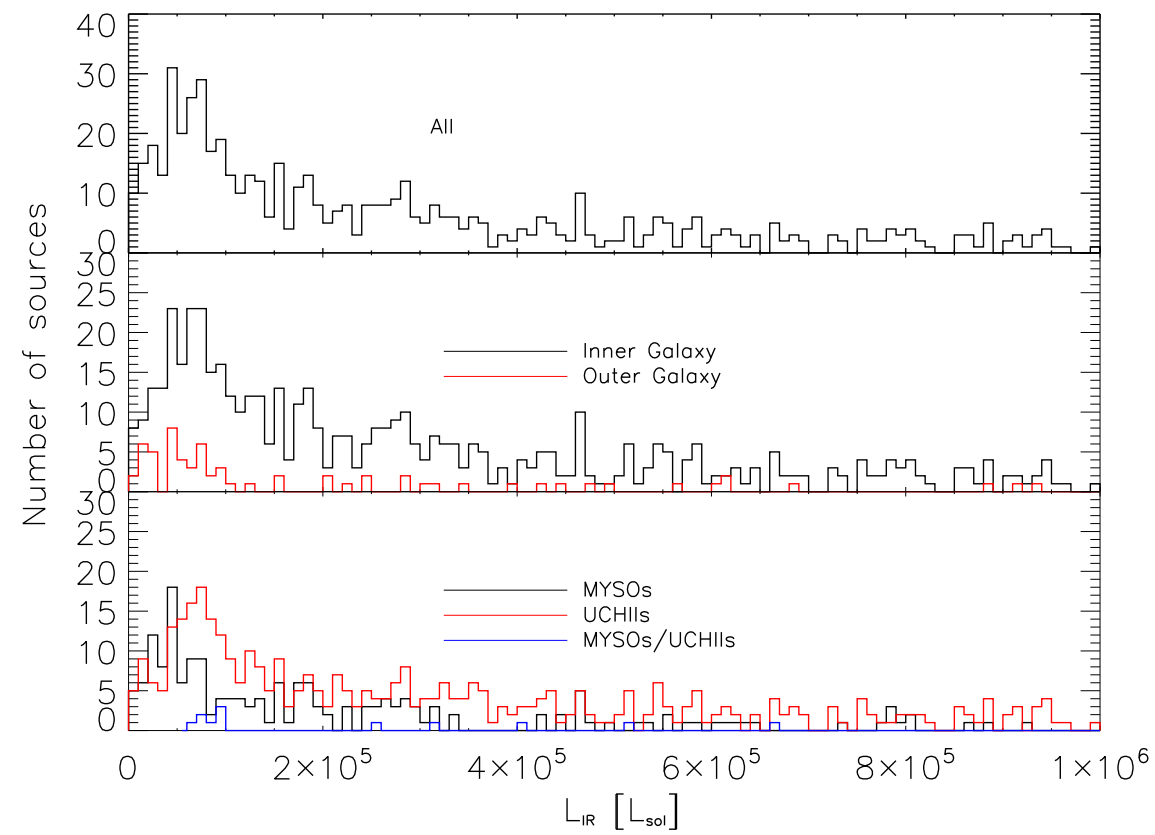

Figure 5. Top panel: overall distribution of luminosities for the complete sample. Middle panel: inner and outer Galaxy luminosity distribution. Bottom panel: MYSOs, UCH II regions, and MYSO/UCH II luminosity distribution.

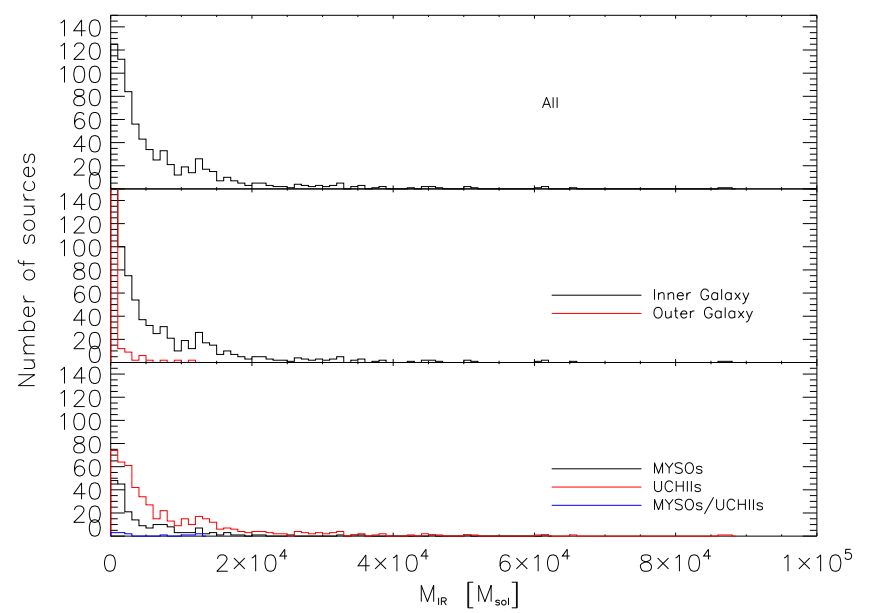

Figure 6. Top panel: overall distribution of mass for the complete sample. Middle panel: inner and outer Galaxy mass distribution. Bottom panel: MYSOs, UCH II regions, and MYSOs/UCH II mass distribution.

process that depends on many parameters. If the determining factors in defining the star formation activity at a given location in the Galaxy were quantities that linearly scale with the Galactocentric radius, such as gas column density and metallicity, we would expect a much tighter correlation. Despite the large scatter in every bin, a global trend emerges from both Figures 8 and 9: the mean luminosity-to-mass ratio decreases toward the outer Galaxy, with a difference between the three innermost bins $\left(2 \mathrm{kpc}<R_{\mathrm{G}}<8 \mathrm{kpc}\right)$ and the three outermost ones ( $\left.8 \mathrm{kpc}<R_{\mathrm{G}}<14 \mathrm{kpc}\right)$ of the order of $53 \%$.

Similarly, if one considers the $L_{\mathrm{IR}} / M_{\mathrm{IR}}$ distributions for the inner-/outer-Galaxy sources, using the same convention for defining the two subsamples described in Section 4.1, we obtain $\overline{\left(L_{\mathrm{IR}, \mathrm{i}} / M_{\mathrm{IR}, \mathrm{i}}\right)}=119.1_{-34.2}^{+177.6} L_{\odot} / M_{\odot}$ $\left(\overline{\left(L_{\mathrm{IR}, \mathrm{i}} / M_{\mathrm{IR}, \mathrm{i}}\right)}=118.9_{-31.9}^{+169.4} L_{\odot} / M_{\odot}\right.$ for the fitted size case $)$

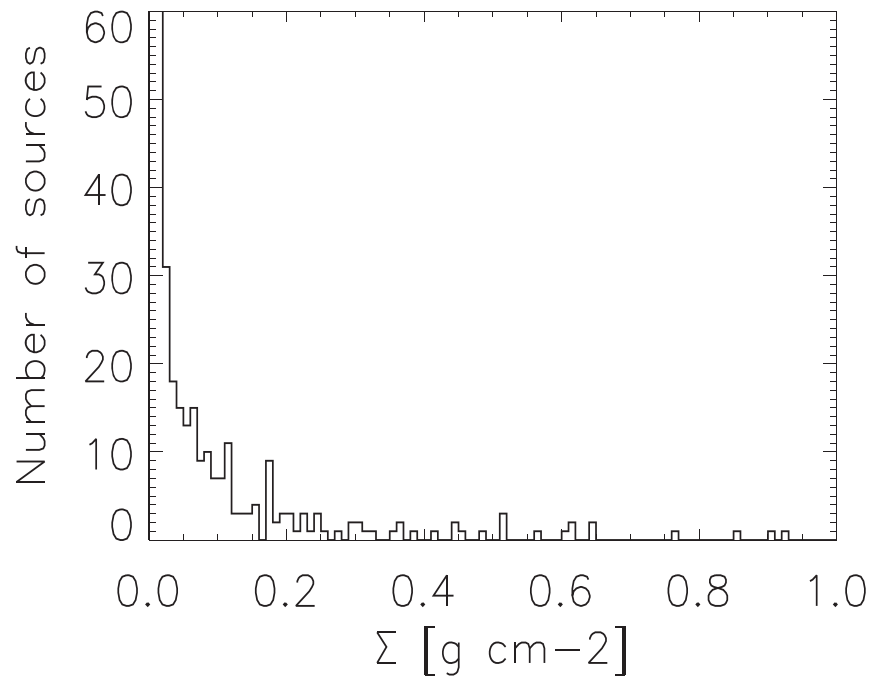

Figure 7. Surface density distribution for the clumps in the complete sample.

and $\overline{\left(L_{\mathrm{IR}, \mathrm{o}} / M_{\mathrm{IR}, \mathrm{o}}\right)}=109.8_{-43.1}^{+1150.8} L_{\odot} / M_{\odot}\left(\overline{\left(L_{\mathrm{IR}, \mathrm{o}} / M_{\mathrm{IR}, \mathrm{o}}\right)}=94.9_{-33.7}^{+135.3}\right.$ $L_{\odot} / M_{\odot}$ for the fitted size case), where $L_{\mathrm{IR}, \mathrm{i}} / M_{\mathrm{IR}, \mathrm{i}}$ and $L_{\mathrm{IR}, \mathrm{o}} / M_{\mathrm{IR}, \mathrm{o}}$ are the IR luminosity to clump mass ratio for the inner- and outer-Galaxy samples, respectively (Figure 10).

If we look closely at Figures 8 and 9 , we notice that the SFE, instead of uniformly decreasing with $R_{\mathrm{G}}$, first increases at small radii $\left(R_{\mathrm{G}}<4 \mathrm{kpc}\right)$ and then peaks at $R_{\mathrm{G}} \sim 5 \mathrm{kpc}$, after which it linearly decreases. This behavior can overall be explained by considering the molecular fraction radial profile as discussed, for instance, in Koda et al. (2016). These authors show that, from a quantitative point of view, the fraction of molecular gas decreases from $100 \%$ near the center of the Galaxy, dropping to $50 \%$ at $6 \mathrm{kpc}$, and decreasing to $10 \%-20 \%$ at $R \sim 8.5 \mathrm{kpc}$. The prominent bump at $\sim 4.5 \mathrm{kpc}$ is due to the bar structure of our Galaxy, with a half-length of 


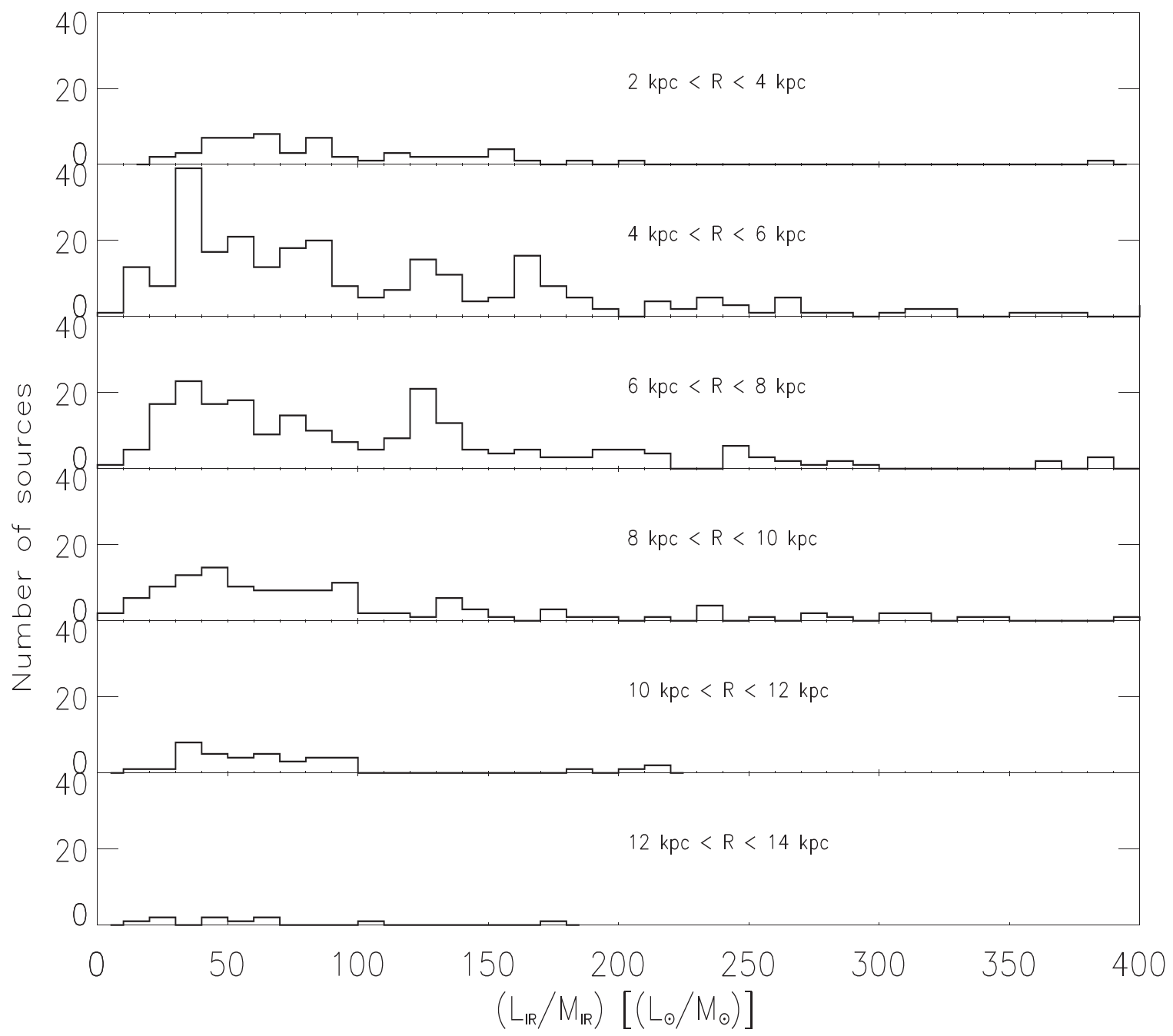

Figure 8. IR luminosity $\left(L_{\mathrm{IR}}\right)$ to dust mass $\left(M_{\mathrm{IR}}\right)$ ratio, $L_{\mathrm{IR}} / M_{\mathrm{IR}}$, as a function of Galactocentric radius, $R_{\mathrm{G}}$.

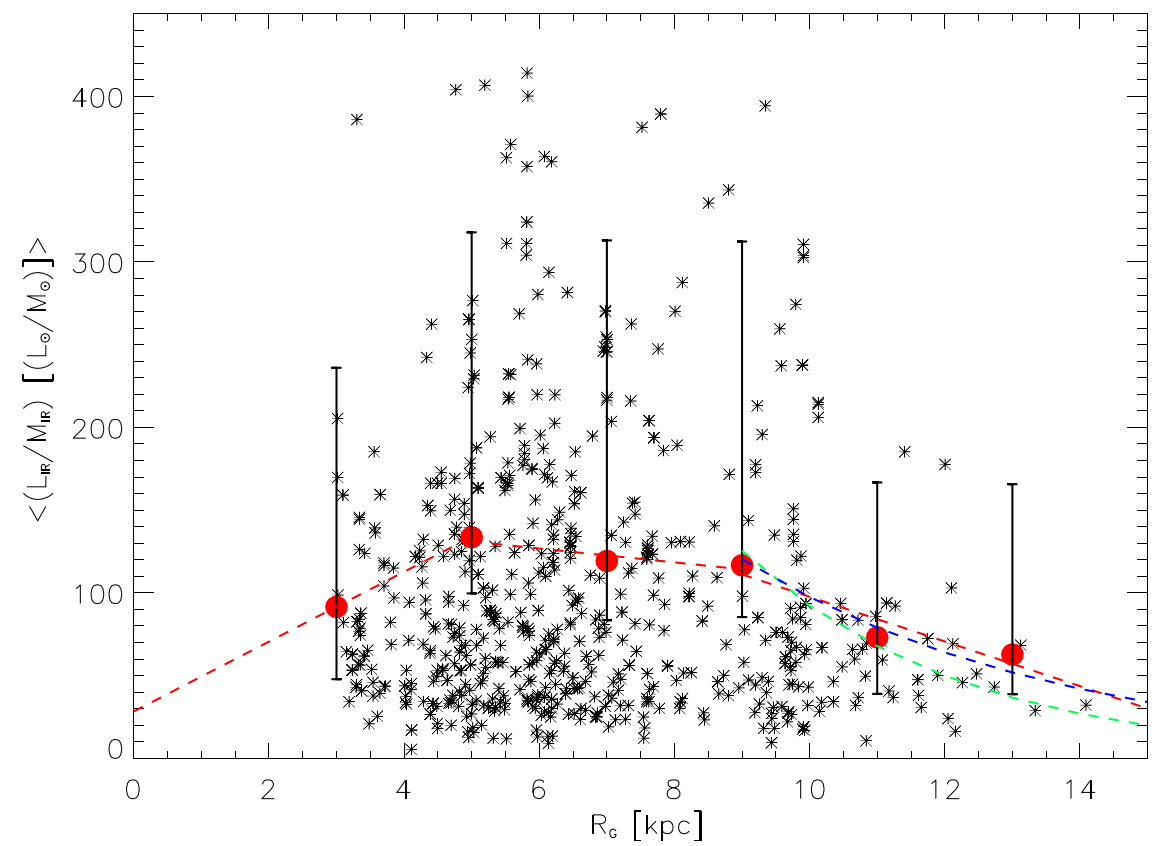

Figure 9. IR luminosity $\left(L_{\mathrm{IR}}\right)$ to dust mass $\left(M_{\mathrm{IR}}\right)$ ratio, $L_{\mathrm{IR}} / M_{\mathrm{IR}}$, as a function of Galactocentric radius, $R_{\mathrm{G}}$. The error bars are the 15.9 th and 84.1 th percentiles. The best fit to the data is shown (red dashed line). Also shown is the exponential decay from Leroy et al. for the case of an optical radius of $13 \mathrm{kpc}$ (green dashed line) and $19 \mathrm{kpc}$ (blue dashed line). The stars represent the individual data points. 
Table 3

Average $L_{\mathrm{IR}} / M_{\mathrm{IR}}$ per Galactocentric Bin

\begin{tabular}{|c|c|c|c|c|c|}
\hline $\begin{array}{l}\text { Bin Center } \\
(\mathrm{kpc})\end{array}$ & No. Sources & $\begin{array}{c}\log \left(L_{\mathrm{IR}} / M_{\mathrm{IR}}\right)(\text { Unresolved }) \\
\log \left(L_{\odot} / M_{\odot}\right)\end{array}$ & $\begin{array}{c}\log \left(L_{\mathrm{IR}} / M_{\mathrm{IR}}\right) \text { (Fitted Sizes) } \\
\log \left(L_{\odot} / M_{\odot}\right)\end{array}$ & $\begin{array}{c}\log \left(L_{\mathrm{IR}} / M_{\mathrm{IR}}\right)(\mathrm{UCH} \text { II }) \\
\log \left(L_{\odot} / M_{\odot}\right)\end{array}$ & $\begin{array}{l}\log \left(L_{\mathrm{IR}} / M_{\mathrm{IR}}\right) \\
\log \left(L_{\odot} / M_{\odot}\right)\end{array}$ \\
\hline 3 & 57 & $91.5_{-43.7}^{+144.5}$ & $83.2_{-44.0}^{+126.2}$ & $81.4_{-39.3}^{+176.8}$ & $83.6_{-44.6}^{+126.2}$ \\
\hline 5 & 274 & $133.6_{-33.9}^{+184.2}$ & $141.8_{-33.1}^{+183.5}$ & $104.6_{-28.6}^{+163.2}$ & $157.9_{-33.2}^{+203.5}$ \\
\hline 7 & 224 & $119.2_{-35.8}^{+193.8}$ & $115.6_{-31.9}^{+174.1}$ & $93.2_{-28.9}^{+152.7}$ & $124.1_{-34.2}^{+180.9}$ \\
\hline 9 & 126 & $116.7_{-31.3}^{+195.6}$ & $107.3_{-30.7}^{+208.9}$ & $88.5_{-23.2}^{+171.6}$ & $113.9_{-31.9}^{+223.9}$ \\
\hline 11 & 39 & $73.1_{-34.2}^{+93.6}$ & $65.5_{-30.7}^{+84.8}$ & $52.3_{-28.3}^{+77.7}$ & $72.9_{-33.7}^{+162.3}$ \\
\hline
\end{tabular}

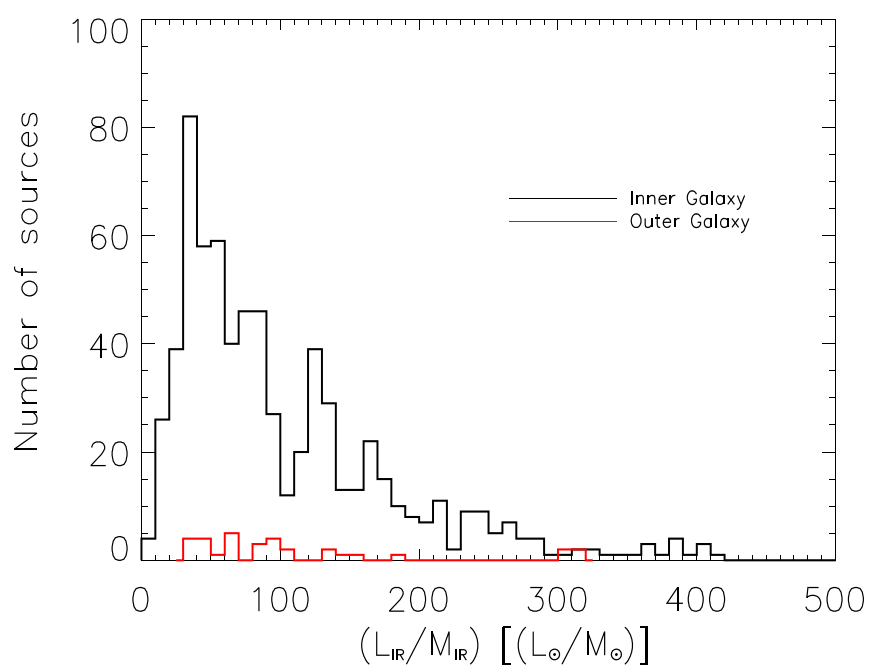

Figure 10. Luminosity-to-mass ratio in the inner (first and fourth) and outer (second and third) Galactic quadrants.

$4.4 \pm 0.5 \mathrm{kpc}$ (Benjamin et al. 2005), and this is associated with bright $\mathrm{CO}$ at the end of the bar, as observed in many external barred spiral systems (e.g., Sheth et al. 2002).

The correlation between SFE and molecular gas is a longstanding problem discussed in the literature, and even more so is the role played by spiral arms. Theoretical models (e.g., Dobbs et al. 2006) predict that spiral arms play a pivotal role in the formation of GMCs and enhance the SFE. However, so far we have been lacking evidence of this. Previous efforts carried out by Moore et al. (2012) in the first Galactic quadrant found that the $L_{\mathrm{IR}} / M_{\mathrm{IR}}$ ratio was relatively flat for the inner $5 \mathrm{kpc}$. Two significant peaks were detected at $\sim 6 \mathrm{kpc}$ and $\sim 8 \mathrm{kpc}$, respectively, but these were attributed to the presence of the star formation complexes W51 and W49. The work of Moore et al. (2012) was later extended by Eden et al. (2013, 2015), who reported no significant variation of the SFE between different arms or the interarm regions. More recently, Urquhart et al. (2018, 2021), in analyzing the ATLASGAL sample in the first and fourth quadrants, found that the SFE as described by the $L_{\mathrm{bol}} / M_{\text {clump }}$ ratio is relatively flat between 2 and $9 \mathrm{kpc}$ when evaluated over kiloparsec scales, although local enhancements can be detected on smaller scales, in agreement with Moore et al. (2012) and Eden et al. (2013, 2015). This body of work led all these authors to conclude that "the spiral arms are principally collecting material together via orbit crowding but there is no evidence that they are playing a role in enhancing the star formation within molecular clouds" (Urquhart et al. 2021, page 12).
We believe that we are detecting correlations of the SFE with Galactocentric distance and with the overall distribution of molecular material, because, for the first time, we are combining three ingredients: (1) a complete sample of objects, (2) the exploration of the Galactic plane as a whole, and (3) the use of data not affected by spatial filtering.

Figure 9 also shows that the peak at $R_{\mathrm{G}} \sim 5 \mathrm{kpc}$ is not followed by a steady decrease in SFE. Instead, the IR luminosity, $L_{\mathrm{IR}}$, to clump mass, $M_{\mathrm{IR}}$, ratio remains almost constant up to $\sim 9 \mathrm{kpc}$. A best fit to $L_{\mathrm{IR}} / M_{\mathrm{IR}}$ as a function of Galactocentric radius gives

$$
L_{\mathrm{IR}} / M_{\mathrm{IR}}= \begin{cases}28.3+21.1 \times R_{\mathrm{G}} & R_{\mathrm{G}}<5 \mathrm{kpc} \\ 152.8-4.2 \times R_{\mathrm{G}} & 5 \mathrm{kpc}<R_{\mathrm{G}}<9 \mathrm{kpc} . \\ 232.6-13.5 \times R_{\mathrm{G}} & R_{\mathrm{G}}>9 \mathrm{kpc}\end{cases}
$$

Importantly, the best-fit solution is obtained by folding in the spread in the data points in each bin. We notice that the two last bins contain only a few data points, i.e., 39 and 11, respectively. However, because the sample is complete, these bins are significant and their weight in the fit is the same as the other bins.

Leroy et al. (2008), based on a study of 23 external spiral and dwarf galaxies, find that in spiral galaxies the SFE appears approximately constant up to the optical radius $\left(r_{25}\right)$, after which it declines exponentially. Taking into account that for our Galaxy the optical radius is estimated to be at $\sim 13-19 \mathrm{kpc}$ (Ruffle et al. 2007; Sale et al. 2010), Leroy et al.'s relation for the Milky Way can be rewritten as

$$
S F E \propto L_{\mathrm{IR}} / M_{\mathrm{IR}}= \begin{cases}\text { const } & R_{\mathrm{G}}<5.6-8.2 \mathrm{kpc} \\ \sim e^{-R_{\mathrm{G}} /(3.25-4.75 \mathrm{kpc})} & R_{\mathrm{G}}>5.6-8.2 \mathrm{kpc}\end{cases}
$$

Figure 9 illustrates the linear fit to the data (Equation 4) and Leroy et al.'s exponential solutions for two values of the optical radius (Equation 5). Leroy et al. point out that the ISM is equal parts $\mathrm{H} \mathrm{I}$ and $\mathrm{H}_{2}$ at $R_{\mathrm{G}}=0.4 \times r_{25} \pm 0.18$. Therefore, their result can be interpreted as a steady decrease of the SFE beyond the $\mathrm{H}$ I-to- $\mathrm{H}_{2}$ transition radius. However, as we see from Figure 9, in the case of our Galaxy, the SFE remains fairly constant up to $R_{\mathrm{G}} \sim 9-10 \mathrm{kpc}$, suggesting either the presence of a significant, previously undetected, molecular material at $R_{\mathrm{G}}>8 \mathrm{kpc}$ and/or that the radial dependence of the SFE might not be simply a function of the $\mathrm{H}$ I-to- $\mathrm{H}_{2}$ transition radius as previously thought.

From the observational point of view, the former finds at least partial confirmation in the hydrogen recombinations lines (H86 $\alpha$ to $\mathrm{H} 93 \alpha$ ) and radio continuum $(9 \mathrm{GHz})$ survey carried 
out by Anderson et al. (2011) with the Green Bank Telescope (GBT). These observations have allowed the discovery, in the first Galactic quadrant, of a new population of $34 \mathrm{H}$ II regions located in the Outer Arm (Bania et al. 2010), at $R_{\mathrm{G}}>8-9 \mathrm{kpc}$. Previously, there were only seven $\mathrm{H}$ II regions known in this area.

Finally, we have investigated variations of the SFE as a function of Galactocentric radius for the two populations of MYSOs and UCH II regions, separately. A behavior similar to the parent population is found for each of these evolutionary stages (see Table 3 ).

\section{Conclusions}

Planck whole-sky submillimeter maps provide an unprecedented opportunity to carry out an unbiased study of the environmental conditions in which massive star formation takes place. Using Planck HFI upper-frequency bands (350, 500 , and $850 \mu \mathrm{m}$ ) complemented by IRAS/IRIS 100, 60 and $25 \mu \mathrm{m}$ bands, we have estimated dust temperatures, luminosities, masses, and surface densities of clumps associated with a complete sample of candidate MYSOs and UCH II regions selected from the RMS survey database.

Exploiting the Planck and IRAS/IRIS full-coverage of the Galactic Plane, we have searched for variations of the clump dust temperatures (warm and cold component) with respect to distance from the Galactic center. We find that the distribution of the cold dust temperature component of the clumps generally reflects the large-scale variation of dust temperature for the ISM along the Galactic plane, that is, massive star complexes at smaller Galactocentric radii have higher temperatures than complexes at larger radii. An opposite trend is found for the warm dust component that appears to increase with distance from the center of the Galaxy, suggesting that the more embedded dust is subject to the local conditions rather than to the ISRF.

We have also explored how the luminosity-to-mass ratio $(L / M)$ associated with the clumps varies from the inner to the outer Galaxy. Our results are consistent with an SFE decreasing from the inner to the outer Galaxy. However, the cutoff radius that identifies the transition between a fairly constant and a declining SFE is at a larger radius $\left(R_{\mathrm{G}} \sim 9 \mathrm{kpc}\right)$ than expected.

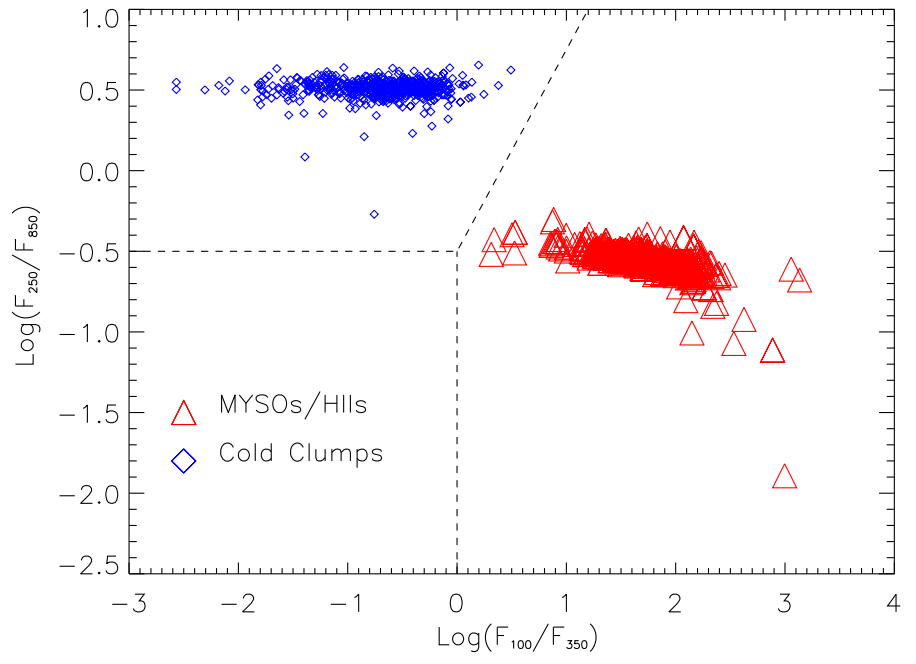

This may have implications for the amount of molecular gas still undetected at $R_{\mathrm{G}}>8 \mathrm{kpc}$.

Finally, we present in the Appendix the color-color and color-magnitude plots of MYSOs and UCH II regions compared to cold clumps from the Planck Catalog of Galactic Cold Clumps (PGCC). These colors will be useful for the identification of sites of massive star formation in future largescale surveys.

This paper is based and made use of information from the Red MSX Source survey database at www.ast.leeds.ac.uk/ RMS which was constructed with support from the Science and Technology Facilities Council of the UK. We thank the anonymous referee for the comments that have greatly improved our work.

\section{Appendix}

The photometric measurements described in Section 4, in addition to allowing us to derive the physical properties of the sources presented above, also make it possible to generate color-color and color-magnitude plots, the purpose of which is to serve as diagnostic tools for the identification of MYSOs and UCH II regions in blind Galactic surveys. To this end, we have performed a random selection from the PGCC (Planck Collaboration et al. 2016b) and compared the colors/ magnitudes of the randomly extracted cold clumps to those of the complete sample of MYSOs and UCH II regions from the RMS database. The PGCC contains 13,188 Galactic cold sources (median temperature between 13 and $14.5 \mathrm{~K}$ ) spread across the whole sky.

Figure 11 shows that the MYSOs and UCH II regions (red triangles) and cold clumps (blue triangles) occupy very distinct regions in the color-color and color-magnitude plots. The MYSOs and UCH II regions are found for:

$-0.5<\log \left(F_{100} / F_{350}\right)<4 .,-2.5<\log \left(F_{250} / F_{850}\right)<0$.

The two populations are characterized by different dust temperatures that cause this segregation: as we have discussed in Section 4.1, there are two temperature dust components of the MYSO and UCH II region SED, one warm, of the order of $30-50 \mathrm{~K}$, and one cold, around $20 \mathrm{~K}$. On the contrary, cold

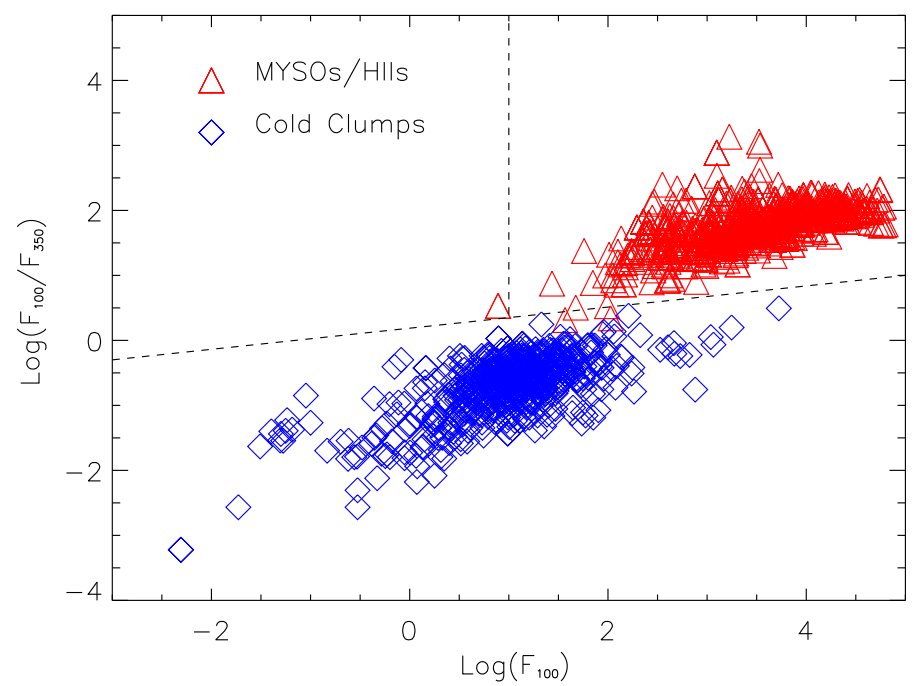

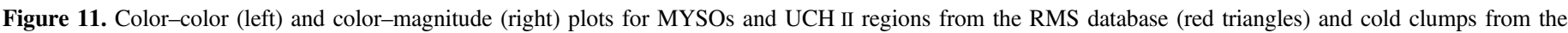
PGCC (blue triangles). 
clumps present only one dust temperature component, i.e., the cold one. This implies that the SED of MYSOs and UCH II regions declines rapidly between 250 and $850 \mu \mathrm{m}$ and between 100 and $350 \mu \mathrm{m}$, while in these wavelength ranges, the spectrum of a cold clump decreases less rapidly $(250<\lambda<$ $750 \mu \mathrm{m})$ or even still rises $(100<\lambda<350 \mu \mathrm{m})$.

\section{ORCID iDs}

R. Paladini (ib https://orcid.org/0000-0002-5158-243X

A. Traficante (D) https://orcid.org/0000-0003-1665-6402

G. Giardino (i) https://orcid.org/0000-0002-9262-7155

D. L. Harrison (iD https://orcid.org/0000-0001-8687-6588

S. Molinari (iD https://orcid.org/0000-0002-9826-7525

\section{References}

Anderson, L. D., Bania, T. M., Balser, D. S., \& Rood, R. T. 2011, ApJS, 194, 32

Bania, T. M., Anderson, L. D., Balser, D. S., \& Rood, R. T. 2010, ApJ, 718, 106

Benjamin, R. A., Churchwell, E., Babler, B. L., et al. 2005, ApJ, 630, 149

Bertoldi, F., \& McKee, C. F. 1992, ApJ, 395, 140

Butler, M. J., \& Tan, J. C. 2012, ApJ, 754, 5

Chini, R., Kreysa, E., Kruegel, E., \& Mezger, P. G. 1986a, A\&A, 166, 8

Chini, R., Kreysa, E., Mezger, P. G., et al. 1986b, A\&A, 154, 8

Chini, R., Kreysa, E., Mezger, P. G., et al. 1986c, A\&A, 157, 1

Chini, R., Krugel, E., \& Wargau, W. 1987, A\&A, 181, 378

Clarke, A. J., Lumsden, S. L., Oudmaijer, R. D., et al. 2006, A\&A, 457, 183

Csengeri, T., Weiss, A., Wyrowski, F., et al. 2016, A\&A, 585, 104

Dame, T. M., Hartmann, D., \& Thaddeus, P. 2001, ApJ, 547, 792

Digel, S. W., Grenier, I. A., Heithausen, A., et al. 1996, ApJ, 463, 609

Dobbs, C. L., Bonnell, I. A., \& Pringle, J. E. 2006, MNRAS, 371, 1663

Eden, D. J., Moore, T. J. T., Morgan, L. K., et al. 2013, MNRAS, 431, 1587

Eden, D. J., Moore, T. J. T., Urquhart, J. S., et al. 2015, MNRAS, 452, 289

Egan, M. P., Price, S. D., \& Kraemer, K. E. 2003, BAAS, 35, 1301

Elmegreen, B. G., \& Hunter, D. A. 2006, ApJ, 636, 712

Giannetti, A., Brand, J., Sanchez-Monge, A., et al. 2013, A\&A, 556, 16

Gorski, K. M., Hivon, E., banday, A. J., et al. 2005, ApJ, 622, 759

Hauser, M. G., Arendt, R. G., Kelsall, T., et al. 1998, ApJ, 508, 25

Heyer, M. H., Brunt, C., Snell, R. L., et al. 1998, ApJS, 115, 241

Juvela, M. J., He, J., Pattle, K., et al. 2018, A\&A, 612, 71

Juvela, M. J., Montillaud, J., Ysard, N., et al. 2013, A\&A, 556, 63

Kennicutt, C. 1998, ApJ, 498, 541

Koda, J., Scoville, N., \& Heyer, M. 2016, ApJ, 823, 76

König, C., Urquhart, J. S., Csengeri, T., et al. 2017, A\&A, 599, A139

Lamarre, J., Puget, J.-P., \& Ade, P. A. R. 2010, A\&A, 520, 9

Leahy, J. P., Bersanelli, M., D’Arcangelo, O., et al. 2010, A\&A, 520, 8

Leroy, A., Walter, F., Brinks, E., et al. 2008, AJ, 136, 2782

Lucas, P. W., Hoare, M. G., Longmore, A., et al. 2008, MNRAS, 391, 136

Lumsden, S. L., Hoare, M. G., Oudmaijer, R. D., \& Richards, D. 2002, MNRAS, 336, 621

Lumsden, S. L., Hoare, M. G., Urquhart, J. S., et al. 2013, ApJS, 208, 11

Mandolesi, N., Bersanelli, M., Butler, R. C., et al. 2010, A\&A, 520, 3

Mathis, J. S., Mezger, P. G., \& Panagia, N. 1983, A\&A, 128, 212

Minniti, D., Lucas, P. W., Emerson, J. P., et al. 2010, NewA, 15, 433

Miville-Deschenes, M.-A., \& Lagasche, G. 2005, ApJS, 157, 302

Miville-Deschenes, M.-A., Murray, N., \& Lee, E. 2017, ApJ, 834, 57

Molinari, S., Pezzuto, S., Cesaroni, R., et al. 2008, A\&A, 481, 345

Moore, T. J. T., Urquhart, J. S., Morgan, L. K., et al. 2012, MNRAS, 426, 701
Mottram, J. C., Hoare, M. G., Urquhart, J. S., et al. 2011, A\&A, 525, 149

Neugebauer, G., Habing, H. J., van Duinen, R., et al. 1984, ApJ, 278, L1

Ossenkopf, V, \& Henning, T 1994, A\&A, 291, 943

Paladini, R., Davies, R. D., \& De Zotti, G. 2004, MNRAS, 347, 237

Paladini, R., Umana, G., Veneziani, M., et al. 2012, ApJ, 760, 149

Planck Collaboration, Adam, R., Ade, P. A. R., et al. 2016a, A\&A, 594, A10

Planck Collaboration, Ade, P. A. R., Aghanim, N., et al. 2011a, A\&A, 536, A1

Planck Collaboration, Ade, P. A. R., Aghanim, N., et al. 2011b, A\&A, 536, A17

Planck Collaboration, Ade, P. A. R., Aghanim, N., et al. 2011c, A\&A, 536, A7

Planck Collaboration, Ade, P. A. R., Aghanim, N., et al. 2016b, A\&A, 594, A28

Planck HFI Core Team, Ade, P. A. R., Aghanim, N., et al. 2011, A\&A, 536, A4

Plume, R., Jaffe, D. T., Evans, N. J., et al. 1997, ApJ, 476, 730

Preibisch, Th., Oseenkopf, V., Yorke, H. W., \& Henning, Th. 1993, A\&A, 279, 577

Povich, M. S., Stone, J. M., Churchwell, E., et al. 2007, ApJ, 660, 346

Reid, M. J., Menten, K. M., Zheng, X. W., et al. 2009, ApJ, 700, 137

Robin, A. C., Creze, M., \& Mohan, V. 1992, ApJL, 400, 25

Robin, A. C., Reyle, C., Derriere, S., \& Picaud, S. 2003, A\&A, 409, 523

Robitaille, T. P., Whitney, B. A., Indebetouw, R., et al. 2006, ApJS, 167, 256

Robitaille, T. P., Whitney, B. A., Indebetouw, R., \& Wood, K. 2007, ApJS, 169,328

Rosset, C., Tristram, M., Ponthieu, N., et al. 2010, A\&A, 520, 13

Ruffle, P. M. E., Millar, T. J., Roberts, H., et al. 2007, ApJ, 671, 1766

Ruphy, S., Robin, A. C., Epchtein, N., et al. 1996, A\&A, 313, 21

Sale, S. E., Drew, J. E., Knigge, C., et al. 2010, MNRAS, 402, 713

Schnee, S., Kauffmann, J., Goodman, A., \& Bertoldi, F. 2007, ApJ, 657, 838

Schuller, F., Menten, K. M., Contreras, Y., et al. 2009, A\&A, 504, 415

Scoville, N. Z., \& Sanders, D. B. 1987, in Interstellar Processes, ed. D. J. Hollenbach \& H. A. Thronson (Dordrecht: D. Reidel), 21

Shaver, P. A., McGee, R. X., Newton, L. M., Danks, A. C., \& Pottasch, S. R. 1983, MNRAS, 204, 53

Sheth, K., Vogel, S. N., Regan, M. W., et al. 2002, AJ, 124, 2581

Skrutskie, M. F., Cutri, R. M., Stiening, R., et al. 2006, AJ, 131, 1163

Solomon, P. M., Rikvolo, A. R., Barrett, J., \& Yahil, A. 1987, ApJ, 319, 730

Tauber, J. A., Mandolesi, N., Puget, J.-P., et al. 2010, A\&A, 520, 1

Traficante, A., Fuller, G. A., Duarte-Cabral, A., et al. 2020, MNRAS, 491,4310

Traficante, A., Fuller, G. A., Smith, R. J., et al. 2018, MNRAS, 473, 4975

Urquhart, J. S., Busfield, A. L., Hoare, M. G., et al. 2007a, A\&A, 461, 11

Urquhart, J. S., Busfield, A. L., Hoare, M. G., et al. 2007b, A\&A, 474, 891

Urquhart, J. S., Figura, C., Cross, J. R., et al. 2021, MNRAS, 500, 3050

Urquhart, J. S., Hoare, M. G., Lumsden, S. L., et al. 2008, in ASP Conf. Ser. 387, Massive Star Formation: Observations Confront Theory, ed. H. Beuther, H. Linz, \& T. Henning (San Francisco, CA: ASP), 381

Urquhart, J. S., Hoare, M. G., Purcell, C. R., et al. 2009, A\&A, 501, 539

Urquhart, J. S., Konig, C., Giannetti, A., et al. 2018, MNRAS, 473, 1059

Urquhart, J. S., Moore, T. J. T., Csengeri, T., et al. 2014, MNRAS, 443, 1555

Urquhart, J. S., Thompson, M. A., Moore, T. J. T., et al. 2013, MNRAS, 435,400

Whitney, B. A., Robitaille, T. P., Indebetouw, R., et al. 2005, in Proc. IAU Symp. 227, Massive Star Birth: A Crossroads of Astrophysics, ed. R. Cesaroni et al. (Cambridge: Cambridge Univ. Press), 206

Williams, J. P., Blitz, L., \& McKee, C. F. 2000, in Protostars and Planets IV, ed. V. Mannings, A. P. Boss, \& S. S. Russell (Tucson, AZ: Univ. Arizona Press), 97

Wright, E. L., Eisenhardt, P. R. M., Mainzer, A. K., et al. 2010, AJ, 140, 1868 Yong, D., Carney, B. W., \& Teixera de Almeida, M. L. 2005, AJ, 130, 597

Zhang, Y., \& Tan, J. C. 2011, ApJ, 733, 55 